OPEN ACCESS

Edited by:

Fei Chen,

Southern University of Science and Technology, China

Reviewed by:

Adriane Ribeiro Teixeira,

Federal University of Rio Grande do

Sul, Brazil

Andrea Canale,

University of Turin, Italy

Judith Eunkyung Cho Lieu, Washington University in St. Louis,

United States

*Correspondence:

Arianna Di Stadio

ariannadistadio@hotmail.com;

Arianna.distadio@unipg.it

Specialty section:

This article was submitted to Auditory Cognitive Neuroscience,

a section of the journa

Frontiers in Neuroscience

Received: 30 March 2020

Accepted: 04 August 2020

Published: 08 September 2020

Citation:

della Volpe A, Ippolito $V$,

Roccamatisi D, Garofalo S,

De Lucia A, Gambacorta V, Longari F, Ricci $G$ and Di Stadio A (2020) Does

Unilateral Hearing Loss Impair Working Memory? An Italian Clinical

Study Comparing Patients With

and Without Hearing Aids.

Front. Neurosci. 14:905.

doi: 10.3389/fnins.2020.00905

\section{Does Unilateral Hearing Loss Impair Working Memory? An Italian Clinical Study Comparing Patients With and Without Hearing Aids}

\author{
Antonio della Volpe', Valentina Ippolito' ${ }^{1}$, Dalila Roccamatisi², Sabina Garofalo1, \\ Antonietta De Lucia1 ${ }^{\text {, Valeria Gambacorta }}{ }^{3}$, Fabrizio Longari ${ }^{3}$, Giampietro Ricci ${ }^{3}$ and \\ Arianna Di Stadio ${ }^{3,4 *}$ \\ 'Otology and Cochlear Implant Unit, Department of Otolaryngology, Santobono-Pausilipon Children's Hospital, Naples, Italy, \\ ${ }^{2}$ Psychology Faculty, Università Telematica Internazionale Uninettuno, Rome, Italy, ${ }^{3}$ Department of Otolaryngology, \\ University of Perugia, Perugia, Italy, ${ }^{4}$ Neuroinflammation Laboratory, Queen Square Neurology, University College London, \\ London, United Kingdom
}

Working memory (WM) function can be reduced in patients suffering from unilateral hearing loss $(\mathrm{UHL})$ and can affect their academic performance. We aimed to compare the WM abilities of three categories of children with UHL: patients implanted with hearing aids (HAs), patients receiving a bone-anchored hearing implant (BAHI), and subjects who did not receive hearing devices. A randomized clinical study, in which 45 children (mean age: 9.5 years) were evaluated by pure tone audiometry (to identify the side and the severity of the UHL), was conducted in a tertiary referral center. Patients were simply randomized into three groups: (1) children without HAs (No-HA group), (2) patients with a (digital) HA (HA group), and (3) children with a BAHI (BAHI group). Their working and short-term memories were studied in both noisy and silent conditions at the recruiting time (TO, baseline) and 6 months after (T1) the treatment. Statistical analyses were performed to analyze the variances between T0 and T1 within each group and between the three groups. The No-HA group improved its T1 WM scores in silence $(p<0.01)$, but not in noise. The HA and BAHI groups showed statistically significant variances of T1 WM in noise ( $p<0.01$ and $p<0.01$, respectively). The HA and BAHI groups did not show statistically significant variances compared to T1. Our results suggest that hearing devices (HA and $B A H I)$ in children with sensorineural UHL (SUHL) can improve WM capacity in noise. We speculate that bilateral hearing capacity might improve the quality of life of this population, especially during everyday activities where noise is present.

Keywords: unilateral hearing loss, hearing aids, speech rehabilitation, working memory, hearing loss, bone anchored hearing device

\section{INTRODUCTION}

The central auditory pathway is bilaterally stimulated by the ears due to crossing fibers, so a unilateral hearing function could modify sound perception (Peterson and Hamel, 2019). When both ears (afferent pathways) work normally (healthy subject), sounds reach the central auditory area reinforced (Popper and Fay, 2019) due to a summation of the auditory stimulus. The 
reinforcement of the stimulus positively affects the left brainresponsible for speech understanding and production (Knösche et al., 2002) - and it allows correct understanding of the meaning of speech and development of normal language skills, especially in children (Di Stadio et al., 2018).

The literature has shown that restoring bilateral hearing functions in children can improve speech perception (Glasberg and Moore, 1989; Mok et al., 2010; Di Stadio and Lazzaro, 2015; Di Stadio et al., 2018), language skills (Lieu et al., 2010; Di Stadio and Lazzaro, 2015; Fitzpatrick et al., 2019), and memory functions (Lyxell et al., 2003; Ning et al., 2013; Di Stadio et al., 2018). The improvement of memory function is related to the increase in attention, and the latter is better when the subject can hear correctly with both ears (Corballis, 2014; Bartolomeo and Seidel Malkinson, 2019). Other studies showed that children with unilateral hearing loss (UHL) can benefit from bone-anchored hearing implant (BAHI) rehabilitation especially at school, as shown by the improvement of their dictation skills (Di Stadio et al., 2018). Dictation skills need to be supported by memory [short-term memory (SM)] functions, so their improvement is directly correlated with the increase of memory performance; the hearing device allowed UHL children to improve their working memory (WM) scores up to the same results as healthy children, and this could explain the observation of Di Stadio et al. (2018).

There is only one study that analyzed the impact of bilateral hearing restoration on the memory performance of UHL children, only investigating patients exclusively treated by BAHI. Even today, there is very little information on the effect of different hearing aids on memory abilities. We therefore aimed to compare the effects of non-use vs. use of hearing aids (HA or BAHI) on memory performance in a sample of children suffering from UHL of varying severity.

\section{MATERIALS AND METHODS}

The study was conducted at the Cochlear Implantation Centre of the Santobono-Pausilipon Children's Hospital in Naples (Italy) from January to September 2019, and it was performed according to Helsinki rules for human study. The protocol was approved by the internal review board (IRB) of the hospital, without issuing an authorization number in respect to national regulations for these types of studies. Before including the children in the study, all parents needed to sign a written consent, in which strengths and weaknesses of each treatment were discussed.

Forty-five children [24 males and 21 females, average age 9.5 years $(S D: 2.8)]$ affected by congenital $[70.8 \%$ of children $(32$ subjects) suffered from HL connexin 26-related, 19 heterozygous (60\%) and 13 homozygous mutation, 25\% from cytomegalovirus (CMV)-related HL, and $4.2 \%$ of mitochondrial disease-related HL] sensorineural UHL (SUHL) that ranged from mild (26$40 \mathrm{~dB})$ to severe $(90 \mathrm{~dB})$ and who presented a normal hearing threshold on the contralateral side were enrolled in the study (Table 1).

All children performed CT scan to evaluate the presence of temporal bone abnormalities; if some abnormalities were identified, the patients were excluded from the study.
The inclusion criteria were first diagnosis of SUHL, patient never rehabilitated by speech therapy and/or hearing device, Italian mother-tongue, and without known/evident intellective deficit.

All patients were tested twice, at baseline (T0) and 6 months after their first control (T1). They underwent a bilateral auditory pure tone test (PTA) at T0 in order to evaluate their hearing capacity and to confirm the presence and the side of the SUHL (details below) and then at T1. A Speech Perception Test (SPT) was also performed at $\mathrm{T} 0$ and $\mathrm{T} 1$ in all children.

Three groups were defined: group 1 (control group) in which patients with SUHL were not treated with hearing aid (No-HA group), group 2 that included patients who were treated with hearing aid (HA), and group 3 in which patients' SUHL was treated by a bone conduction system (BAHI group).

The children were randomized by computer software to be assigned to one of the three groups and then to be treated as indicated by the relative group. The computer generated the random numbers that were used for the simple randomization. For a correct distribution in each group, the patients were separately randomized according to their HL severity; first, patients with mild SUHL were randomized, then the ones with more severe forms up to the ones with profound SUHL. Despite this initial randomization, we then revised the assignation of the children to one or another group to avoid undertreatment or overtreatment of SUHL, so we cannot define this study as a real randomized study.

The randomization by different severities of SUHL was possible because the children had one ear with normal hearing threshold. Children with mild SUHL had an HL in the range in which BAHI could be an effective treatment.

Patients in group 1 were untreated for speech rehab, while patients in groups 2 and 3 were trained by a speech therapist to optimize the use of their hearing aid. Patients with BAHI performed speech rehab once their prosthesis was osteointegrated. However, regardless of which group they were assigned to, all children underwent cognitive and learning rehabilitation.

All patients in group 2 used Xceed Play $1 \mathrm{BTE} \mathrm{SP}^{1}$ as hearing device. This digital device, specifically intended for children, is designed for treatment of sensorineural hearing loss (SNHL) in

${ }^{1}$ www.oticon.com

TABLE 1 | The table summarizes demographic details of the patients included in the study.

\begin{tabular}{lccc}
\hline & No-HA & HA & BAHI \\
\hline Age & $10 \pm 3.43(\mathrm{SD})$ & $10 \pm 2.41(\mathrm{SD})$ & $9 \pm 2.17(\mathrm{SD})$ \\
Gender & 13 patients: & 16 patients: & 16 patients: \\
& 7 male- 6 female & 7 male- 9 female & 10 male- 6 female \\
Hearing loss PTAv & $63.5 \pm 8.18(\mathrm{SD})$ & $63.5 \pm 12.6(\mathrm{SD})$ & $63.7 \pm 10(\mathrm{SD})$ \\
TO* & & &
\end{tabular}

"Please see additional details of patients' auditory thresholds Table 4. No-HA, no hearing aids; HA, hearing aids; $B A H I$, bone anchored hearing implants; $S D$, standard deviation; PTAv, pure tone average. 
the severity range between 30 and $110 \mathrm{~dB}$. The device covers a frequency range between 100 and 6,500 HZ, has an OSPL90 with maximum peak at $143 \mathrm{~dB}$ SPL and $135 \mathrm{~dB}$ SPL at 1,600 Hz, and allows a maximum of $83 \mathrm{~dB}$ at gain peak and $75 \mathrm{~dB}$ at 1,600 Hz. Its harmonic distortion at $70 \mathrm{~dB}$ SPL is $4 \%$ at $500 \mathrm{~Hz},<2 \%$ at $800 \mathrm{~Hz}$, and $<2 \%$ at $1,600 \mathrm{~Hz}$. The device entry noise level was $18 \mathrm{~dB}$ SPL omni and $32 \mathrm{~dB}$ SPL directional. Finally, it has an integrated receiver with a power of $2.4 \mathrm{GHz}$ and is FM compatible. The hearing aids were used to compensate the hearing deficit. Hearing aids had to be worn all day from wake-up to bed time.

Patients in group 3 were implanted with BAHA $^{\circledR 2}$. The BAHA $^{\circledR}$ was implanted percutaneously as previously described by Ricci et al. (2019) by the same surgeon with decades of experience in hearing devices. In this group, the BAHI was used as a CROS device.

The memory abilities were evaluated in all children at T0 before randomization both in silent and in noisy conditions (T0) and at T1-6 months after the hearing rehabilitation or the application of a hearing prosthesis. This 6-month follow-up was necessary to allow a correct osteointegration of the BAHI (3 months in all our patients) and adequate speech rehab once the prosthesis was implanted. A memory evaluation included testing both the WM and the SM, as detailed below. All tests were conducted by a speech therapist and a psychologist together; both professionals had more than 10 years of experience in their respective field.

\section{Details on Clinical Investigation Pure Tone Audiometry}

The auditory tests were performed differently at T0 and T1. At T0, the patient was tested by earphones in a silent cabin. At T1, children were analyzed in free-field using speakers because they were wearing a hearing prosthesis. The T1 auditory test was performed with the patient seated in the center of the cabin where two speakers at $45^{\circ}$ were placed one on each side of the patient. A pulse-tone was emitted by the speaker on the side of the ear that had to be tested while a white-noise sound (masquerading sound) was sent by an insert located on the opposite side. This procedure was performed bilaterally. The sound stimulation started from $10 \mathrm{~dB}$, with increases of $10 \mathrm{~dB}$ and decreases of $5 \mathrm{~dB}$, to confirm the sound perception. The impulse for each frequency tested (250, $500,1,000,2,000,4,000,6,000$, and $8,000 \mathrm{~Hz}$ ) was sent to the studied ear three times, following the method described above. The masking signal (white noise range frequency $2-16 \mathrm{kHz}$ ) was sent at $50 \mathrm{~dB}$ and the testing signal at 20-30 dB on the ear in which we were evaluating the hearing threshold. Pure tone average (PTAv) was calculated by summing the results of pure tone audiometry (PTA) thresholds at 500, 1,000, and 2,000 Hz and dividing the total by 3 .

\section{Speech Perception Test}

The test was performed in the same conditions as PTA. A list of simple words (Table 2) was used in place of the pulse tone and the cocktail party sound (several adult talkers) replaced the white noise sound (masquerading sound). The masking signal

${ }^{2}$ www.cochlear.com was sent at $50 \mathrm{~dB}$, and the testing signal at $20-30 \mathrm{~dB}$ on the ear in which we were evaluating the hearing threshold. The test was performed with the same procedure described for PTA. SPT was measured with an average percentage, with values from $0 \%$ total absence of perception up to $>95 \%$ indicating perception of all proposed words.

\section{Working Memory Testing}

The WM actively manages received information. It allows us to understand the meaning of a sentence even if the meaning of each individual word is not known (Baddeley, 1981). Evaluation of the WM was performed with PROMEA battery tests. Subjects were asked to repeat one meaningless word at a time; the word (bisyllabic to multisyllabic) consisted of an alternation of vowels and consonants with sounds very phonologically similar to known (existing) Italian words (Vicari, 2007). A "non-word" sentence is a sentence composed of pseudo-words, which are words that have a sound similar to words in the (Italian) language but which, in fact, have no meaning [e.g., "sasta" sounds similar to "pasta" (Vicari, 2007) but does not mean anything]. A subject with a properly functioning WM will correct the non-word "sasta" to "pasta," indicating an understanding of the meaning of the sentence as a whole. All tests were performed in both silent and noisy (cocktail party noise) conditions (Hutcherson et al., 1979). Test scores were calculated by dividing the number of correct answers by the total number of questions, which was 39, as suggested by Vicari in the book Tests for Memory and Learning in Childhood. The memory test was standardized for children aged 5-11 years (Bisiacchi et al., 2005; Vicari, 2007). The final score was calculated in percentages as follows: $0-25 \%$ severe WM deficit; $26-50 \%$ moderate WM deficit; $51-75 \%$ adequate WM; and $76-100 \%$ excellent WM.

\section{Short-Term Memory Testing}

The SM stores information for a brief period of time, typically 10-15 s (Baddeley and Patterson, 1971). Evaluation of the SM was performed by asking the subjects to repeat the words exactly as they were heard. This test was performed under silent conditions only, as noise may affect SM function and impair

TABLE 2 | The table shows the list of Italian words used to perform speech perception test in Italian.

\begin{tabular}{llll}
\hline List of words used for speech perception test in children & \\
\hline mamma & camion & lupo & albero \\
scuola & letto & spada & quadro \\
nonno & frigorifero & occhio & chiodo \\
orologio & fiori & cesto & stelle \\
uno & giornale & televisione & regina \\
macchina & sedia & occhiali & treno \\
pipa & famiglia & farfalla & due \\
forchetta & limoni & cane & acqua \\
casa & gatto & bagno & bicicletta \\
capelli & sci & barca & pantaloni
\end{tabular}

This words' list is specifically designed for children and it was the only used for testing all patients. 
the subject's ability to hear a non-word. We chose repetition of phonologically similar words (similarity effect) to evaluate and stimulate the phonological loop, the efficiency of which is strongly related to the auditory function. The test was performed by presenting six blocks (Glasberg and Moore, 1989; Knösche et al., 2002; Di Stadio and Lazzaro, 2015; Di Stadio et al., 2018; Peterson and Hamel, 2019; Popper and Fay, 2019) of words not commonly used (low-use frequency) in sequence. Each block of words contained a sequence of bisyllabic words, i.e., "luna-topo," which were presented to the patients five times consecutively at intervals of 2-3 s. The test started by proposing a list of two words, then three, four, etc. If the patient correctly repeated $3 / 5$ sequences of heard words, the examiner went ahead to the next span level. The answers to each block identify a different level of span, from 1 to 6 . Final scores of span level could range from 1 to 6 , where scores between 1 and 3 indicated an SM deficit, and scores $\geq 4$ indicated normal SM function. This SM test was standardized for children aged between 5 and 11 years (Bisiacchi et al., 2005).

\section{Data Analysis}

Because all patients were analyzed after 6 months from their first control (T0), the three groups did not present statistically significant differences in observation time; the variable "time" was not considered due to this reason. All WM scores (groups 1, 2, and 3 ) collected at T0 in noisy and in silent conditions and at T1 in the same conditions were compared using a one-way ANOVA test and by Holm-Bonferroni method. The same analysis was performed to compare SM scores at T0 and T1 in the three groups (groups 1, 2, and 3). We also analyzed the difference between T0 and $\mathrm{T} 1$ considering $\mathrm{T} 1$ as dependent variable by using a twotailed $t$-test. Our sample included patients affected by SUHL on different sides, so we also evaluated the differences between the right and left side. For all tests, the level of significance was set to 0.05 . The statistical analysis was performed with Stata ${ }^{\circledR}$ and was supervised by an expert in biostatistics and epidemiology.

\section{RESULTS}

\section{General}

The time elapsed (days) between the first (T0) and the second (T1) follow-up in the three groups was: No-HA 180 (SD: 3.8), HA 180.3 (SD: 3.4), and BAHI 180.6 (SD: 3.7).

Group 1 (No-HA) included four patients with mild HL, five with moderate $\mathrm{HL}$, three suffering from moderate to severe $\mathrm{HL}$, and one with severe SUHL. In group 2 (HA), eight patients suffered from mild SUHL, five with a moderate form, two affected by moderate-severe SUHL and one with severe SUHL. In group 3 (BAHI), we observed three patients affected by mild SUHL, two with a moderate form, two with moderate-severe SUHL, and nine with severe SUHL.

Table 3 shows the results of PTA and SPT at T0 and T1 in the three groups.

Twenty-five subjects [15 males and 10 females, mean age: 9.3 years $(S D: 2.8)$ ] suffered from right-sided SUHL, while 20 subjects were affected by left-sided SUHL [five males and seven
TABLE 3 | The table shows the results of PTA and SPT in the three groups at $\mathrm{TO}$ and $\mathrm{T} 1$.

\begin{tabular}{lccc}
\hline & No-HA & HA & BAHI \\
\hline PTAv T0 & $63.5 \pm 8.18(\mathrm{Cl}$ & $63.5 \pm 12.6(\mathrm{Cl}$ & $63.7 \pm 10(\mathrm{Cl} 95 \%:$ \\
& $95 \%: 55-80)$ & $95 \%: 45-90)$ & $55-90)$ \\
PTAv T1 & $57 \pm 8.2(\mathrm{Cl} 95 \%:$ & $23.2 \pm 6.5(\mathrm{Cl} 95 \%:$ & $22 \pm 3.3(\mathrm{Cl} 95 \%:$ \\
& $45-70)$ & $15-35)$ & $20-30)$ \\
SPT T0 & $45 \% \pm 9(\mathrm{Cl} 95 \%:$ & $45.7 \% \pm 12(\mathrm{Cl} 95 \%:$ & $42.7 \% \pm 3(\mathrm{Cl} 95 \%:$ \\
& $30-65)$ & $35-70)$ & $40-45)$ \\
SPT T1 & $52 \% \pm 8(\mathrm{Cl} 95 \%:$ & $91.5 \% \pm 8(\mathrm{Cl} 95 \%:$ & $93 \% \pm 6(\mathrm{Cl} 95 \%:$ \\
& $40-65)$ & $75-100)$ & $80-100)$ \\
\hline
\end{tabular}

No-HA, no hearing aids; HA, hearing aids; $B A H I$, bone anchored hearing implant; PTAv, pure tone average; SPT, speech perception test.

females, mean age: 10 years (SD: 2.8)]. A statistically significant variance was identified between the WM scores in noise and silence at T0 in all three groups $(p<0.00001$; BH subgroup $1: p<0.01$; subgroup 2: $p<0.01$; subgroup 3: $p<0.01)$; no statistically significant differences were identified between the groups at T0 (Table 4 and Figure 1).

No statistically significant variance was observed in silence between the WM scores at T0 and $\mathrm{T} 1$ in group 1 (No-HA; $\mathrm{BH}$ : $p=0.3$ ) or in noise (Figures 2, 3). Group 2 (HA) presented statistically significant variances in noise comparing T0 and T1 (HB: $p<0.01$ ), but no statistically significant differences were observed in silence. A similar finding was observed in group 3 (BAHI; HB: $p<0.01$; Figures 2, 3). Statistically significant differences in the WM scores were observed at T1 (silence and noise) between patients in group 1 (No-HA) and subjects with hearing devices (HA and BAHI; HB: $p<0.01$ and $p<0.01$, respectively; Figures 2, 3). No statistically significant differences were observed in groups 2 and 3 between the WM scores at T1. The side of SUHL (right vs. left) did not affect the WM scores at T0 and T1 (no statistically significant result). No statistically significant variances were observed by comparing the SM scores at T0 and T1 between groups and within each group (Figure 4).

The analysis of data, considering $\mathrm{T} 1$ as a dependent variable of T0, showed in the No-HA group a statistically significant difference in the WM scores both in noise ( $t$-test: $p=0.01$ ) and silence ( $t$-test: $p=0.01$ ), but not statistically significant values in SM ( $t$-test: $p=1)$. HA and BAHI groups presented statistically significant differences in the WM scores in noise, respectively $p<0.00001$ and $p<0.00001$. No statistically significant differences were observed in silence in HA ( $t$-test: $p=0.09$ ) and BAHI ( $t$-test: $p=0.05)$ patients. SM values were not statistically significantly different in both groups (HA: $t$-test: $p=1$ and BAHI: $p=1$ ) (Table 5).

Finally, no statistically significant variances were observed in the SM scores (T0 and T1) between right- and left-sided SUHL.

\section{DISCUSSION}

Our study showed that the restoration of a bilateral hearing function in UHL subjects could improve WM functions, especially in noisy condition, and that this improvement was 
TABLE 4 | Summary of PTAv at T0, WM at T0 (silence and noise), SM at T0, WM at T1 (silence and noise), and SM a T1.

\begin{tabular}{|c|c|c|c|c|c|c|c|c|}
\hline Group & Side of UHL & Hearing thresholds PTAv TO (dB) & WM T0 noise & WM T1 noise & WM T0 silence & WM T1 silence & SM TO & SM T1 \\
\hline No-HA & Right & 55 & $0 \%$ & $0 \%$ & $91.6 \%$ & $92 \% \%$ & 4 & 4 \\
\hline No-HA & Right & 80 & $0 \%$ & $0 \%$ & $100 \%$ & $100 \%$ & 2 & 2 \\
\hline No-HA & Right & 35 & $66 \%$ & $66 \%$ & $87 \%$ & $90 \%$ & 4 & 4 \\
\hline No-HA & Right & 40 & $29.1 \%$ & $30 \%$ & $100 \%$ & $100 \%$ & 5 & 5 \\
\hline No-HA & Right & 60 & $15 \%$ & $15 \%$ & $92.3 \%$ & $92.5 \%$ & 4 & 4 \\
\hline No-HA & Right & 55 & $8 \%$ & $10 \%$ & $92 \%$ & $92 \%$ & 4 & 4 \\
\hline No-HA & Right & 70 & $8 \%$ & $9 \%$ & $80 \%$ & $80 \%$ & 2 & 2 \\
\hline No-HA & Right & 55 & $4 \%$ & $8 \%$ & $92 \%$ & $92 \%$ & 4 & 4 \\
\hline No-HA & Right & 40 & $30 \%$ & $30 \%$ & $100 \%$ & $100 \%$ & 4 & 4 \\
\hline$H A$ & Right & 55 & $41.6 \%$ & $80 \%$ & $100 \%$ & $100 \%$ & 3 & 3 \\
\hline$H A$ & Right & 90 & $0 \%$ & $46 \%$ & $92 \%$ & $92 \%$ & 4 & 4 \\
\hline$H A$ & Right & 35 & $45 \%$ & $83 \%$ & $87 \%$ & $87 \%$ & 4 & 4 \\
\hline$H A$ & Right & 40 & $37.5 \%$ & $70 \%$ & $100 \%$ & $100 \%$ & 4 & 4 \\
\hline$H A$ & Right & 70 & $12 \%$ & $88 \%$ & $90 \%$ & $90 \%$ & 3 & 3 \\
\hline$H A$ & Right & 40 & $30 \%$ & $84.6 \%$ & $92.3 \%$ & $92.3 \%$ & 4 & 4 \\
\hline$H A$ & Right & 35 & $41 \%$ & $100 \%$ & $91.6 \%$ & $91.6 \%$ & 4 & 4 \\
\hline$B A H I$ & Right & 55 & $0 \%$ & $80 \%$ & $80 \%$ & $85 \%$ & 2 & 3 \\
\hline$B A H I$ & Right & 40 & $41.6 \%$ & $95 \%$ & $41.6 \%$ & $41.6 \%$ & 3 & 3 \\
\hline$B A H I$ & Right & 60 & $12.5 \%$ & $33 \%$ & $100 \%$ & $100 \%$ & 4 & 4 \\
\hline$B A H I$ & Right & 85 & $37.5 \%$ & $79.4 \%$ & $50 \%$ & $60 \%$ & 4 & 4 \\
\hline$B A H I$ & Right & 90 & $6 \%$ & $92.3 \%$ & $92.3 \%$ & $92.3 \%$ & 2 & 4 \\
\hline$B A H I$ & Right & 75 & $41 \%$ & $89.4 \%$ & $76.9 \%$ & $78 \%$ & 3 & 3 \\
\hline$B A H I$ & Right & 90 & $0 \%$ & $60 \%$ & $13 \%$ & $15 \%$ & 3 & 3 \\
\hline$B A H I$ & Right & 90 & $46 \%$ & $79.4 \%$ & $82 \%$ & $83 \%$ & 3 & 3 \\
\hline$B A H I$ & Right & 90 & $0 \%$ & $33 \%$ & $61 \%$ & $62 \%$ & 4 & 4 \\
\hline No-HA & Left & 35 & $0 \%$ & $0 \%$ & $63 \%$ & $64 \%$ & 3 & 3 \\
\hline No-HA & Left & 55 & $0 \%$ & $0 \%$ & $70.8 \%$ & $71 \%$ & 4 & 4 \\
\hline No-HA & Left & 65 & $8 \%$ & $10 \%$ & $80 \%$ & $82 \%$ & 2 & 2 \\
\hline No-HA & Left & 35 & $0 \%$ & $0 \%$ & $63 \%$ & $64 \%$ & 3 & 3 \\
\hline No-HA & Left & 50 & $4 \%$ & $5 \%$ & $92 \%$ & $92 \%$ & 4 & 4 \\
\hline No-HA & Left & 55 & $0 \%$ & $4 \%$ & $70.8 \%$ & $72 \%$ & 4 & 4 \\
\hline$H A$ & Left & 35 & $64 \%$ & $100 \%$ & $94.8 \%$ & $95 \%$ & 4 & 4 \\
\hline$H A$ & Left & 55 & $2 \%$ & $61 \%$ & $92 \%$ & $92 \%$ & 4 & 4 \\
\hline$H A$ & Left & 40 & $2 \%$ & $66 \%$ & $87.50 \%$ & $89 \%$ & 4 & 4 \\
\hline$H A$ & Left & 60 & ०\% & $30 \%$ & $48 \%$ & $50 \%$ & 3 & 3 \\
\hline$H A$ & Left & 55 & $0 \%$ & $95 \%$ & $97.4 \%$ & $98 \%$ & 4 & 4 \\
\hline$H A$ & Left & 45 & $37.5 \%$ & $79 \%$ & $70 \%$ & $70 \%$ & 4 & 4 \\
\hline$H A$ & Left & 40 & $38 \%$ & $58 \%$ & $95 \%$ & $95 \%$ & 5 & 5 \\
\hline$B A H I$ & Left & 35 & $72 \%$ & $100 \%$ & $100 \%$ & $100 \%$ & 3 & 3 \\
\hline$B A H I$ & Left & 45 & $16 \%$ & $62.5 \%$ & $100 \%$ & $100 \%$ & 4 & 4 \\
\hline$B A H I$ & Left & 90 & $0 \%$ & $60 \%$ & $13 \%$ & $15 \%$ & 3 & 3 \\
\hline$B A H I$ & Left & 65 & $41 \%$ & $89.4 \%$ & $76.9 \%$ & $76.9 \%$ & 3 & 3 \\
\hline$B A H I$ & Left & 75 & $2 \%$ & $70 \%$ & $100 \%$ & $100 \%$ & 4 & 4 \\
\hline$B A H I$ & Left & 35 & $72 \%$ & $100 \%$ & $100 \%$ & $100 \%$ & 3 & 3 \\
\hline$B A H I$ & Left & 80 & $2 \%$ & $70 \%$ & $100 \%$ & $100 \%$ & 4 & 4 \\
\hline
\end{tabular}

No-HA, no hearing aids; HA, digital hearing aids; BAHI, bone anchored hearing implant; WM, working memory; SM, short-memory; PTAv, pure tone average.

independent of the device used to rehab the auditory capacity, of the side affected by HL and of the severity of HL.

These current results reinforce other recent observations (Di Stadio et al., 2018), in which the authors showed that children with UHL rehabilitated by BAHI could improve their WM capacities up to the levels observed in healthy children. In addition, these positive results were more consistent when the memory functions were tested in noisy condition.

The analyses on $\mathrm{T} 1$ as dependent variable of T0 confirmed that HA and BAHI had improved the scores of WM in noise but not in silence and that the scores of SM remained substantially the 
TABLE 5 | The table summarizes SD and mean in each group of WM at T0 and T1 both in silence and noise, and SM at T0 and T1.

\begin{tabular}{lcccrrr}
\hline Group & WM T0 (\%) noise & WM T1 (\%) noise & WM T0 (\%) silence & WM T1 (\%) silence & SM T0 & SM T1 \\
\hline No-HA & $10.2 \pm 0.2$ & $12.4 \pm 0.2$ & $86.2 \pm 0.1$ & $84.5 \pm 0.1$ & $3.5 \pm 0.9$ \\
HA & $21.3 \pm 0.2$ & $74 \pm 0.2$ & $86 \pm 0.1$ & $88 \pm 0.1$ & $3.5 \pm 0.9$ \\
BAHI & $25 \pm 0.3$ & $73 \pm 0.2$ & $74.5 \pm 0.3$ & $76 \pm 0.3$ & $3.8 \pm 0.5$ & $3.2 \pm 0.7$
\end{tabular}

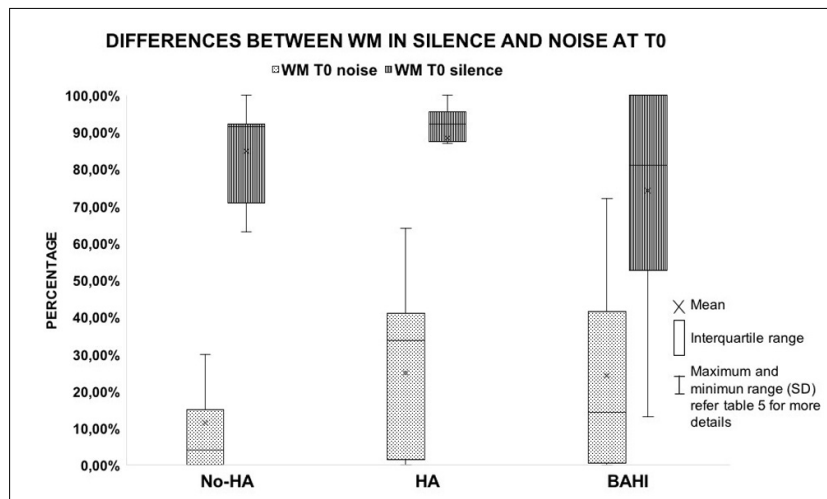

FIGURE 1 | The image shows the differences observed in the working memory score between the test performed in silent and noisy conditions at T0 in the three groups. All the horizontal lines in the box divide II and III quartiles.

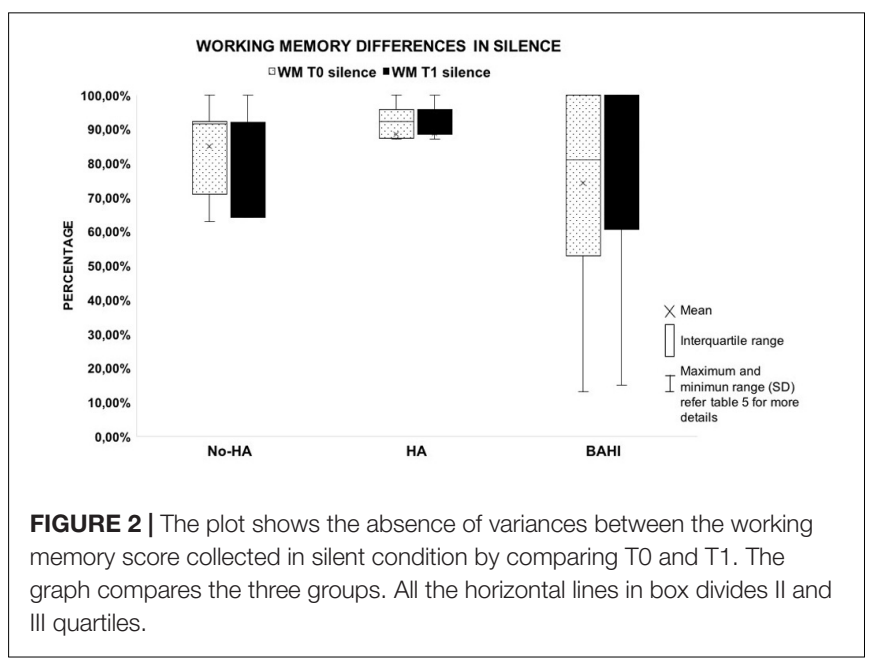

same at the two follow-ups (T0-baseline and T1-6 months after baseline). Interesting data were that No-HA children statistically improved their WM scores in silence but not in noise; in the latter, we instead observed a statistically significant worsening of the WM capacities. We speculated that the difference observed in noise scores between the three groups could be related to three factors: (1) use of hearing device, (2) speech rehabilitation, and (3) cognitive and learning rehabilitation. In fact, in the NoHA group, the cognitive and learning rehab alone negatively impacted on the WM abilities in noise as shown by the statistically significant worsening of the scores that we observed.

We also noted that, regardless of the severity of UHL, patients with hearing devices improved their PTA and SPT on comparing T0 and T1 (Table 3); this positive effect of hearing rehabilitation

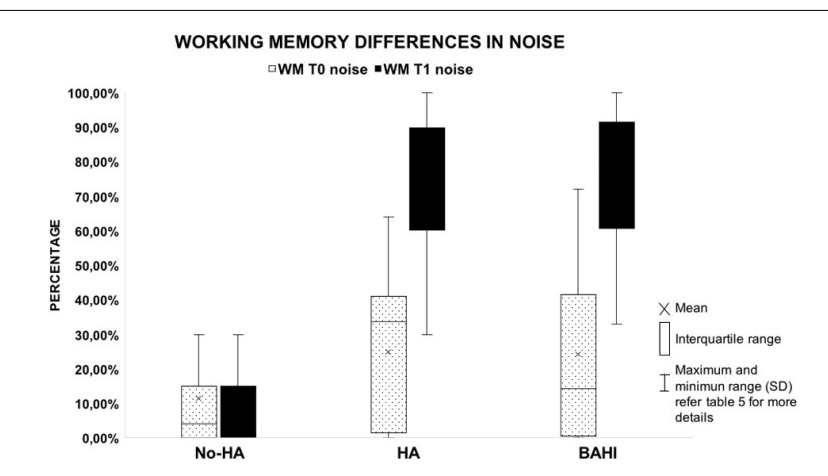

FIGURE 3 | The graph illustrates the recovery of working memory in the noisy condition. Despite the fact that patients with no hearing aids $(\mathrm{HA})$ improved their scores, the results failed to reach statistical significance. All the horizontal lines in the box divides II and III quartiles.

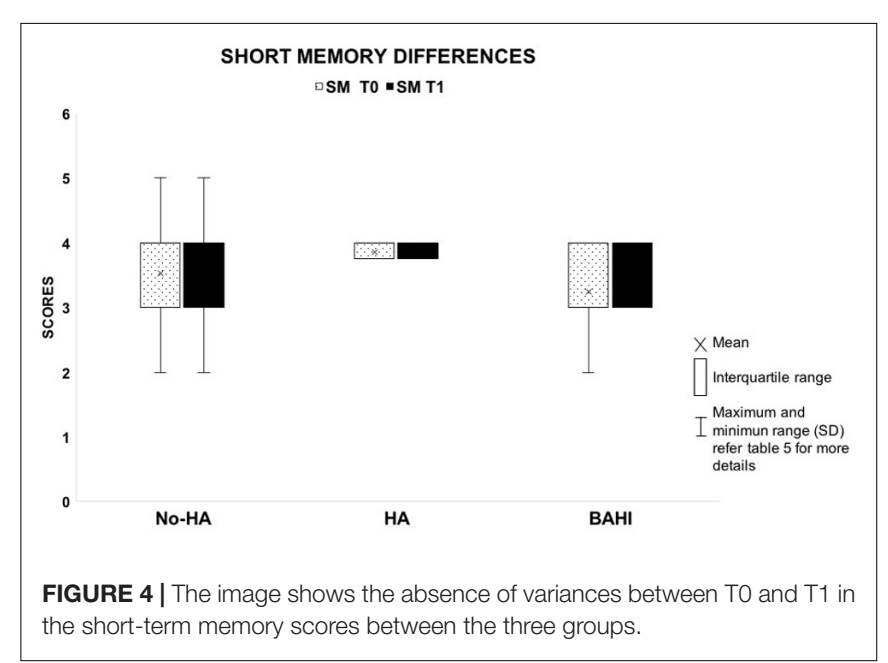

on speech perception in children with UHL-regardless of HL severity-was already shown by Purcell et al. (2016). In addition to the hearing device, also the speech rehabilitation performed by the children in groups 2 and 3 could have been helpful in improving PTA and SPT capacities.

Bishop et al. (2017) showed that the increase in speech perception could improve WM performance; the observed memory recovery could be related not only to the improved speech perception but also to an increased perception of external stimuli (Arndt et al., 2017) since both conditions stimulate memory functions (Cardon and Sharma, 2013). The mechanism which drives the improvement of WM is based on the amelioration of attention (Cherubini, 2012); the restoration of bilateral hearing allowed the patient to capture information 
more correctly and to better select which of these could be relevant or not (Cherubini, 2012). Thanks to the better hearing capacity, patients were able to strategically shift the focus of attention between maintenance and processing over time and in the most appropriate ways (Cherubini, 2012). However, we cannot exclude that speech rehabilitation also had positively impacted on WM function by partially influencing the results observed. In fact, both hearing restoration and rehabilitation performed by the children could have stimulated their brain plasticity as previously shown by Kolb et al. (2017). These authors focused their analyses on brain plasticity, and they found that children's brains were extremely sensitive to changes in external stimuli (Kolb et al., 2017), so the restoration of bilateral hearing function had positively impacted on their brain maturation (Cardon and Sharma, 2013; Kolb et al., 2017). Furthermore, as previously shown by Pisoni (1973), the correct identification of words positively impacts on memory functions.

Although both are very efficient in rehabilitating auditory functions, the hearing devices that we used in our study worked in different ways. The digital device improved the sound perception by increasing the volume of the incoming sound (Levitt, 2007); the BAHI, on the other hand, stimulated the cochlea by a vibratory stimulation activating cochlea hair cells and by exciting the basilar membrane through the inertia of the inner ear fluid and the compression and expansion of the inner ear space (Stenfelt, 2015). In addition, the BAHI could retrogradely stimulate the perilymph through the vibration of cerebrospinal fluid (CSF) (Sohmer et al., 2000). Nevertheless, our results showed that, regardless of the hearing device, bilateral hearing function improved the memory performances. However, considering that children with BAHI underwent delayed speech rehab compared to patients with a hearing device, we could affirm that this type of hearing rehabilitation allowed better auditory performance; in fact, children achieved an improvement of WM performance as good as the one observed in patients with hearing aids but with fewer speech rehabilitation training sessions.

The benefits achievable by bilateral hearing functions have already been described (Krishnan and Van Hyfte, 2016); this restoration impacts positively on memory functions (McCoy et al., 2005; Härkönen et al., 2015; Di Stadio et al., 2018) and improves word recollection performances (Skinner, 1957) by helping school-age children in the execution of certain tasks [e.g., dictation (Di Stadio et al., 2018)]. On the contrary, patients affected by untreated UHL can suffer from behavioral consequences (Brookhouser et al., 1991) and present unsatisfactory academic performances (Dancer et al., 1995). A correct hearing function, especially in noise (silence is rarely present in children's classrooms), is fundamental to avoid developmental delays (Brookhouser et al., 1991; Härkönen et al., 2015).

Although there is still some controversy on the use of hearing devices to support children with UHL (McKay et al., 2008; Bagatto, 2020)-some authors have indicated that a reduction of hearing performance in noise may be related to distraction on the side in which UHL was treated by hearing device (Lewis et al., 2016)-our results suggest that bilateral hearing functions could be necessary to improve the memory abilities of UHL children, particularly in everyday life rich in surrounding noise (Di Stadio et al., 2020). In fact, regardless of the severity of their HL, all our children presented a worsening of WM scores comparing their answers in silence and noise at T0, meaning that noise negatively impacts on memory performances. At the end of our study, we noted the improvement of the WM scores in all children (regardless of the severity of their HL), although the highest gains were identified in the most severe cases of UHL (T0 vs. T1). This result is in contrast with the idea that a hearing device in UHL can negatively impact on a child's performance (Bagatto, 2020).

Based on our results, we suggest that a hearing device should always be used to avoid impairment of scholastic performance, regardless of the severity and the side of the HL.

An important aspect to emphasize was the strong impact that speech rehabilitation had on maximizing the benefit of the hearing device. The speech therapist helped patients with hearing devices to focus their attention on what could be better perceived by wearing a prosthesis. This aspect was very helpful in better managing the distress related to wearing the device and improving patient compliance. The speech rehabilitation helped subjects to correctly focus their attention on the new external stimuli. The speech therapist not only increased the compliance of children in using the prosthesis, but she also supported parents in the management of this new situation, i.e., answering the questions related to the use of the device. The speech training was useful for children and parents/guardians. The latter, by understanding the efficacy of the prosthesis, strongly supported their children's hearing aid compliance.

The study presents some limitations. Firstly, the right-sided UHL group was slightly larger than the left-sided UHL group, and this may induce bias due to the unbalanced distribution of subjects. Secondly, the tests used to evaluate speech perception and memory functions were designed exclusively for Italian speakers, and other authors could obtain different results using other tests. In addition, we were focused on memory only and the attention of the patients could only be indirectly evaluated by looking at the results of the memory tests. Furthermore, we did not analyze if sound localization could impact on memory function, although we did not find differences comparing patients with hearing device on the right side with the ones in the left one. Additional studies investigating the effect of sound localization on memory function would be necessary.

Our patients' selection, in which we excluded patients affected by temporal bone abnormalities, might have determined a bias; in addition, the study has been performed on a small sample and other researchers might obtain different results studying a larger sample. In fact, our population of children could not reflect the broader universe of children with UHL.

Another limitation, related to an ethical reason, was the slight unbalance between untreated patients (No-HA: 13 subjects) and patients treated with hearing aids (HA: 16 people and BAHI: 16 patients). Moreover, because of ethical and clinical reasons, group 3 (BAHI) included more patients with severe SUHL than 
the other two groups; in fact, restoration of an efficient hearing threshold was the main goal of this treatment. Finally, because of the use of a hearing device, auditory tests were differently performed at T0 (no hearing device) and T1 (wear hearing device), and this difference in tests might have an impact on the results of the study. However, this limitation is present in all studies of this type.

\section{CONCLUSION}

Hearing restoration via hearing aids/BAHI or hearing therapy should be suggested as the treatment of patients suffering from UHL, given that bilateral hearing stimulation may positively impact on the WM processes, especially in noisy conditions (Di Stadio et al., 2020). In fact, non-rehabilitated UHL could lead to a reduction in memory performance during school activities (rich in noise) which could negatively impact on academic performance. We suggest rehabilitation by hearing device for all children affected by UHL, regardless of the severity of their HL, to avoid possible deficiencies in school performances.

\section{DATA AVAILABILITY STATEMENT}

The raw data supporting the conclusions of this article will be made available by the authors, without undue reservation.

\section{REFERENCES}

Arndt, S., Laszig, R., Aschendorff, A., Hassepass, F., Beck, R., and Wesarg, T. (2017). Cochlear implant treatment of patients with single-sided deafness or asymmetric hearing loss. HNO 65, 98-108. doi: 10.1007/s00106-016-0297-295

Baddeley, A. (1981). The concept of working memory: a view of its current state and probable future development. Cognition 10, 17-23.

Baddeley, A. D., and Patterson, K. (1971). The relation between long-term and short-term memory. Br. Med. Bull. 27, 237-242.

Bagatto, M. (2020). Audiological considerations for managing mild bilateral or unilateral hearing loss in infants and young children. Lang. Speech Hear. Serv. Sch. 51, 68-73. doi: 10.1044/2019_LSHSS-OCHL-19-0025

Bartolomeo, P., and Seidel Malkinson, T. (2019). Hemispheric lateralization of attention processes in the human brain. Curr. Opin. Psychol. 29, 90-96. doi: 10.1016/j.copsyc.2018.12.023

Bishop, C. E., Hamadain, E., Galster, J. A., Johnson, M. F., Spankovich, C., and Windmill, I. (2017). Outcomes of hearing aid use by individuals with unilateral sensorineural hearing loss (USNHL). J. Am. Acad. Audiol. 28, 941-949. doi: 10.3766/jaaa.17049

Bisiacchi, P. S., Cendron, M., Gugliotta, M., Tressoldi, P. E., and Vio, C. (2005). BVN 5-11 - Batteria di Valutazione Neuropsicologica per l'età Evolutiva. Las Vegas: Erikson.

Brookhouser, P. E., Worthington, D. W., and Kelly, W. J. (1991). Unilateral hearing loss in children. Laryngoscope 101, 1264-1272. doi: 10.1002/lary.5541011202

Cardon, G., and Sharma, A. (2013). Central auditory maturation and behavioral outcome in children with auditory neuropathy spectrum disorder who use cochlear implants. Int. J. Audiol. 52, 577-586. doi: 10.3109/14992027.2013. 799786

Cherubini, P. (2012). Psicologia generale. Cort. Raffael. 2012, 214-220.

Corballis, M. C. (2014). Left brain, right brain: facts and fantasies. PLoS Biol. 12:e1001767. doi: 10.1371/journal.pbio.1001767

Dancer, J., Burl, N. T., and Waters, S. (1995). Effects of unilateral hearing loss on teacher responses to the SIFTER. Am. Ann. Deaf. 140, 291-294.

\section{ETHICS STATEMENT}

The studies involving human participants were reviewed and approved by Santobono-Posilipon IRB. Written informed consent to participate in this study was provided by the participants' legal guardian/next of kin.

\section{AUTHOR CONTRIBUTIONS}

ADS contributed to the study design, analysis of data, interpretation of data, state of conclusion, supervision of the study, and article writing. AV contributed to the analysis of data, state of conclusion, and data collection and provided substantial contribution in article writing. VI contributed to the data collection and interpretation of data and provided supporting contribution in article writing. SG and ADL contributed to the data collection and data interpretation. VG and FL contributed to supporting data collection. GR contributed to criticism and provided supporting contribution in writing. All authors contributed to the article and approved the submitted version.

\section{ACKNOWLEDGMENTS}

Special thanks to the parents who allowed inclusion of their children in this clinical trial. Thanks to Dr. Massimo Ralli for his suggestions.

Di Stadio, A., Dipietro, L., De Lucia, A., Ippolito, V., Ishai, R., and Garofalo, S. (2020). Novel bone conduction hearing system may improve memory function in children with single side hearing loss: a case-control study. Int. J. Adv. Oto. $16,158-164$.

Di Stadio, A., Dipietro, L., Toffano, R., Burgio, F., De Lucia, A., Ippolito, V., et al. (2018). Working memory function in children with single side deafness using a bone-anchored hearing implant: a case-control study. Audiol. Neurootol. 23, 238-244. doi: 10.1159/000493722

Di Stadio, A., and Lazzaro, D. (2015). DAAR ${ }^{\circledR}$ : new digital software for prosody’s study in subject with monolateral cochlear implant. Prevent. Res. 4, 84-90.

Fitzpatrick, E. M., Gaboury, I., Durieux-Smith, A., Coyle, D., Whittingham, J., and Nassrallah, F. (2019). Auditory and language outcomes in children with unilateral hearing loss. Hear. Res. 372, 42-51. doi: 10.1016/j.heares.2018.03.015

Glasberg, B. R., and Moore, B. C. (1989). Psychoacoustic abilities of subjects with unilateral and bilateral cochlear hearing impairments and their relationship to the ability to understand speech. Scand. Audiol. 32, $1-25$.

Härkönen, K., Kivekäs, I., Rautiainen, M., Kotti, V., Sivonen, V., and Vasama, J. P. (2015). Sequential bilateral cochlear implantation improves working performance, quality of life, and quality of hearing. Acta Otolaryngol. 135, 440-446. doi: 10.3109/00016489.2014.990056

Hutcherson, R. W., Dirks, D. D., and Morgan, D. E. (1979). Evaluation of the speech perception in noise (SPIN) test. Otolaryngol. Head Neck Surg. 87, 239-245.

Knösche, T. R., Lattner, S., Maess, B., Schauer, M., and Friederici, A. D. (2002). Early parallel processing of auditory word and voice information. Neuroimage 17, 1493-1503.

Kolb, B., Harker, A., and Gibb, R. (2017). Principles of plasticity in the developing brain. Dev. Med. Child. Neurol. 59, 1218-1223. doi: 10.1111/dmcn.13546

Krishnan, L. A., and Van Hyfte, S. (2016). Management of unilateral hearing loss. Int. J. Pediatr. Otorhinolaryngol. 88, 63-73. doi: 10.1016/j.ijporl.2016.06.048

Levitt, H. (2007). A historical perspective on digital hearing AIDS: how digital technology has changed modern hearing AIDS. Trends Amplif. 11, 7-24. doi: $10.1177 / 108471380629800$ 
Lewis, D., Schmid, K., O’Leary, S., Spalding, J., Heinrichs-Graham, E., and High, R. (2016). Effects of noise on speech recognition and listening effort in children with normal hearing and children with mild bilateral or unilateral hearing loss. J. Speech Lang. Hear Res. 59, 1218-1232. doi: 10.1044/2016_JSLHR-H-15-0207

Lieu, J. E., Tye-Murray, N., Karzon, R. K., and Piccirillo, J. F. (2010). Unilateral hearing loss is associated with worse speech-language scores in children. Pediatrics 125, 1348-1355. doi: 10.1542/peds.2009-2448

Lyxell, B., Andersson, U., Borg, E., and Ohlsson, I. S. (2003). Working-memory capacity and phonological processing in deafened adults and individuals with a severe hearing impairment. Int. J. Audiol. 42, S86-S89.

McCoy, S. L., Tun, P. A., Cox, L. C., Colangelo, M., Stewart, R. A., and Wingfield, A. (2005). Hearing loss and perceptual effort: downstream effects on older adults' memory for speech. Q. J. Exp. Psychol. A 58, 22-33.

McKay, S., Gravel, J. S., and Tharpe, A. M. (2008). Amplification considerations for children with minimal or mild bilateral hearing loss and unilateral hearing loss. Trends Amplif. 12, 43-54. doi: 10.1177/1084713807313570

Mok, M., Galvin, K. L., Dowell, R. C., and McKay, C. M. (2010). Speech perception benefit for children with a cochlear implant and a hearing aid in opposite ears and children with bilateral cochlear implants. Audiol. Neurootol. 15, 44-56. doi: $10.1159 / 000219487$

Ning, H., Ng, E., Rudner, M., Lunner, T., Syskind, P. M., and Rönnberg, J. (2013). Effects of noise and working memory capacity on memory processing of speech for hearing-aid users. Intern. J. Audiol. 7, 433-441. doi: 10.3109/14992027.2013. 776181

Peterson, D. C., and Hamel, R. N. (2019). Neuroanatomy, Auditory Pathway. StatPearls: Treasure Island, FL.

Pisoni, D. B. (1973). Auditory and phonetic memory codes in the discrimination of consonants and vowels. Percept. Psychophys. 13, 253-260.

Popper, A. N., and Fay, R. R. (2019). The Mammalian Auditory Pathway: Neurophysiology. Switzerland: Springer Nature.
Purcell, P. L., Jones-Goodrich, R., Wisneski, M., Edwards, T. C., and Sie, K. C. Y. (2016). Hearing devices for children with unilateral hearing loss: patient- and parent-reported perspectives. Intern J. Pediatr. Otorhinol. 90, 43-48. doi: 10. 1016/j.ijporl.2016.08.029

Ricci, G., Di Stadio, A., Gambacorta, V., and della Volpe, A. (2019). "Congenital aural atresia: hearing rehabilitation by bone-anchored hearing implant (BAHI)," in Advances in Rehabilitation of Hearing Loss, eds D. Zanetti and F. D. Berardino (London: IntechOpen). doi: 10.5772/intechopen. 88201

Skinner, B. F. (1957). Verbal Behavior. La Jolla, CA: Copley Publishing Group.

Sohmer, H., Freeman, S., Geal-Dor, M., Adelman, C., and Savion, I. (2000). Bone conduction experiments in humans - a fluid pathway from bone to ear. Hear. Res. 146, 81-88.

Stenfelt, S. (2015). Inner ear contribution to bone conduction hearing in the human. Hear. Res. 329, 41-51. doi: 10.1016/j.heares.2014.12.003

Vicari, S. (2007). Prove di Memoria e Apprendimento per l'Età Evolutiva. Florence: Giunti.

Conflict of Interest: The authors declare that the research was conducted in the absence of any commercial or financial relationships that could be construed as a potential conflict of interest.

Copyright (c) 2020 della Volpe, Ippolito, Roccamatisi, Garofalo, De Lucia, Gambacorta, Longari, Ricci and Di Stadio. This is an open-access article distributed under the terms of the Creative Commons Attribution License (CC BY). The use, distribution or reproduction in other forums is permitted, provided the original author(s) and the copyright owner(s) are credited and that the original publication in this journal is cited, in accordance with accepted academic practice. No use, distribution or reproduction is permitted which does not comply with these terms. 